\title{
A Hybrid Model for Emergence of Skilled Agent Specialization with Continuous Demand
}

\author{
Saurabh Singh \\ School of Computer Science \\ University of Windsor \\ Windsor, Canada
}

\author{
Nayan M. Shah \\ Dept. of Applied Mathematics \\ Indian Institute of Technology \\ $(\mathrm{BHU})$, Varanasi, India
}

\author{
Ziad Kobti \\ School of Computer Science \\ University of Windsor \\ Windsor, Canada
}

\begin{abstract}
In this paper, we study the effect of demand on specialization of skilled agents by modifying the earlier hybrid model which is based on the well-known Genetic Threshold Model (GTM) and Social Inhibition Model (SIM). We improve the agent specialization or division of labor (DOL) and also the quality of work (QOW) by introducing a new concept of varying the demand on a smooth curve and compare our results with the previous models.
\end{abstract}

\section{General Terms}

Multi Agent based systems, Modeling, Artificial Intelligence

\section{Keywords}

Division of labor, quality of work, agent specialization

\section{INTRODUCTION}

In artificial society, agents are able to reason about the environment to maximize the performance to achieve their individual goals. There are many ways by which agents can improve their task performance and increase the productivity. Some of the approaches are: (i) Agents can learn from their past experience and improve, (ii) Agents can interact within the same group or across the other groups to discuss about the demand and supply of a particular task, and (iii) Agents can choose to pick tasks on the basis of skill inheritance from the family.

Specialization is allocating a disproportionate amount of a resource to one task compared to other available tasks. In a population of heterogeneous individuals, it is often the case that these individuals possess different aptitudes for available tasks. Individuals increase their productivity by enhancing their specialization in communities of mutual interest, whereby other individuals are also trying to maximize their productivity in relation to competitors [1].

Division of labor or specialization is one of the primary attributes of sociality. Caste and specialization have been the focus of the study of the organization of insect societies for more than fifty years. Indeed, the description and analysis of task allocation between colony members are fundamental to understand the organization of a complex biological system whose functioning depends upon the behavioral integration of a potentially large number of individuals or agents. The advantage of specialization by individuals within groups is also considered to be of overwhelming importance in many of the major transitions in the evolution of life [2].

The evolutionary transition from solitary organisms to highly integrated societies composed of individual organisms (e.g. ant colonies, termite colonies and certain bees and wasps) is also associated with efficiencies that accrue from a division of labor and task specialization. Social insect colonies have been compared to factories within fortresses [3] and there are many different tasks that agents (workers) must perform, from building the nest and guarding the colony to tending the queen, rearing many different stages of brood, and feeding and grooming one another. Division of labor, where different units within a system perform different tasks, is a recurrent property of association of multiple entities and a hallmark of social living. This fundamental property has been described across a diversity of social taxa, from simple to complex groups. However, empirical evidence suggests that division of labor in social groups increases with increasing group size [45]. Larger groups size is phylogenetically correlated with more complex and derived sociality, as seen recurrently within the social insects [3], suggesting that the pattern may reflect selection acting to increase individual specialization. There is also a trend towards increased division of labor during social ontogeny, as social groups grow from few individuals to many, as shown in [6-7]. A model providing insight into possible mechanisms contributing to division of labor was given in [8] and it was shown that an increase in division of labor could parallel an increase in group size directly via the distribution of thresholds within groups and indirectly via by-products of increased group size (i.e. task number and demand).

There are several different ways to cause the emergence of specialization within a complex system. The agents may choose their specialization or it may be assigned as is the case in caste system. Several factors including genetic, social and economic considerations affect the choice of specialization [9], but no approach can fully explain specialization in a complex system [10]. These different approaches work with the limitation of their own assumptions and contexts thus making it difficult to compare results across these different approaches [11].

Several genetic models have been proposed for the study of specialization. The most widely used are the response thresholds model. The thresholds model presents a certain level of stimulus for each task at which an individual will choose to specialize in that task [12]. In the threshold model, agents by default perform no tasks. It means if there is no stimulus for any of the possible tasks, then individual will do nothing [9]. Agents will also perform no tasks if none of the stimuli for all available tasks fail to cross its response threshold [9]. The threshold varies between agents. In some approaches, performing a task causes the thresholds level for that task to decrease, while not performing the task will lead to the thresholds level increasing [12].

Social inhibition models also play an important role in the emergence of agent's specialization. According to this approach agents choose their specialization, they notify other agents that they have done so, reducing the desire of others to choose this specialization. 
Division of labor (DOL) and quality of work (QOW) are the two main components which are discussed as a function of discretely, randomly and continuously varying demands in this paper. The DOL statistic measures the degree to which different individuals within the group specialize on different tasks and the degree to which each individual is specialist [8]. Quality of work (QOW) measure the average amount of skill used in performing a task. The higher values of DOL and QOW are indicative of increase in specialization among the agents and that the task was performed by a more skilled agent.

Cockburn and Kobti [13] created a weight allocated social inhibition approach whereby more skilled agents inhibit the desire of less skilled agents to perform a task. This approach drives agents toward tasks where they have comparative advantages. This leads to an increase in specialization within the population. Though their approach was inspired by social insects, this approach is entirely applicable to agents in other domains. Combining the Genetic Threshold Model (GTM), and the Social Inhibition Model (SIM), they proposed a model aiming to increase the effect of agent skill on task choice when agents possess different aptitudes for tasks. Their model increased the level of quality of work (QOW), but with the side effect of reduced levels of specialization. In their model, agents choose randomly among tasks with surpassed threshold or be inactive if no such task exists. They supposed that each time a task $i$ is performed by an individual, the stimulus intensity $S_{i}$ is decreased by an amount $\alpha=3$. For each time step, the level of stimulus $S_{i}$ associated with task $i$ is increased by $\beta_{i}=\alpha N / T$, where $\mathrm{N}$ is the group size (number of individuals) and $T$ is the task number. The reduced demand consequent with increased group size should positively affect DOL as shown by [8]. So they had incorporated demand $\delta$ in the expression for $\beta_{i}$ as given by equation (5). But they fixed the demand for all tasks thus the rate of stimulus regeneration is identical for all tasks and does not vary with time.

The decreased level of specialization in [13] and the identical rate of stimulus regeneration in [8] motivated us for the present work. In this paper, the model [13] is modified assuming the same characteristics of agents; varying skill levels for each task and the ability to divide resources among tasks. Further, the effect of demand $\delta$ on division of labor (DOL) and quality of work (QOW) is incorporated; a feature missing in [13] but taken into consideration by [8] while analyzing the emergence of increased DOL as a function of group size by taking $\delta=0.7,0.9,1.0$ and 1.1. Demand represents the total colony effort required to complete all tasks relative to the available total effort from workers. The paper analyzes the effect of demand on DOL as well as QOW by (i) taking discrete values of $\delta$ same as in [8], (ii) chooses it randomly in $(0.1,1.1)$ and (iii) let $\delta$ varies continuously through a smooth curve whose profile is given in Figure 2. In the next section, a brief description of the model [13] for continuity and readability of the paper is given.

\section{HYBRID MODEL}

As this model is a modification of the one proposed in [13], the agents will have all the properties of their model like agent attributes, its inhibition, its interaction and its attribute updates. Let $\mathrm{T}$ denote a set of tasks i.e. each element $i \in T$, is a task to be performed by an agent. Each agent has a level of skill $S k_{a}(i)$ associated with each task $i$. The skill level may be dynamic or static and is quantifiable and monotonic, i.e. $S k_{a}(i)>S k_{b}(i)$ means that agent $a$, is more skilled than agent $b$ for task $i$. The strength of inhibition of an agent towards other agents depends upon the skill level of the agent. For each agent $A g$, we have an $A L L O C$ set [13], where $e_{i} \in A L L O C \Rightarrow$ there is a task $i$ in $T_{A g}$ with weight $e_{i}$ allocated to the task $i$, where $T_{A g}$ is the set of tasks available to the agent $A g$. Similarly $R_{A g}$, is the resource available to $\mathrm{Ag}$ to do the tasks in $T_{\mathrm{Ag}}$

Task weights in ALLOC are relative, hence for a given task $i$, the amount of $\mathrm{R}_{\mathrm{Ag}}$ to be allocated to the task $i$ is:

$$
\frac{e_{i}}{S(A L L O C)} \times S\left(R_{A g}\right)
$$

where $S(A L L O C)$ is the sum of all elements in $A L L O C$ and $S\left(R_{A g}\right)$ refers to the total amount of resource available. A task having a weight of 0 will result in the task being allocated none of $R_{A g}$. It will be assumed, without loss of generality, the resource $R$ refers to the time for the rest of the paper. They also normalize the weights in $A L L O C$ such that $S(A L L O C)$ is always equal to 1. Each agent has the following three attributes for all tasks $i \in T$ : (i) Allocation set $A L L O C$, (ii) A skill set $S K I L L=\left\{s_{i}\right\}$ and (iii) A set $P O D S=\left\{p_{i}\right\}$.

For a task $i \in T$, the elements, $e_{i} \in A L L O C$, $s_{i} \in S K I L L$ represent, respectively, the fraction of time the agent will spend and the skill of the agent to perform the task $i$. For $p_{i} \in P O D S, p_{i}$ is a 3 -tuple (A, SA, I), where A represents the activator store for the agent, SA is the level of self-activation, and I is the inhibition store for the agent. The agent will increase or decrease the weight of the associated task depending upon whether $\mathrm{A}+\mathrm{SA}$ is positive or negative respectively. The idea behind self-activation is the inclination of an agent to perform more of the task at which they are best. This value should be large enough that it will allow an isolated agent to specialize over a long period of time, but it should also be small enough that it doesn't overwhelm the social pressure created by stronger competitors. When two agents $A g_{1}$ and $A g_{2}$ interact, for a task $i \in T$, we obtain the values of their PODS for that task $i$. The interaction will decrease the value of A in their respective PODS by the other agent's I, whereas each agent will increase it's A value by its I. Agents will update their allocation based on each task pod. Given an allocation $e_{i}$ and $\operatorname{pod}(a, s, x)$ for a task $i, e_{i}$ will be updated as: $e_{i}=e_{i}+a+s$ i.e. the amount of selfactivator $S$ and activator $a$ is added to the current weight. After all task weights are updated for an agent, the values are again normalized, resulting in the sum of all weights being 1 . Cockburn and Kobti[13], used two equally weighted drivers for changing agent's thresholds: (i) A genetic pull towards performing the task at which the agent is most skilled and (ii) competition from the other agents.

They used the following formula for the genetic pull: 


$$
M T \times\left[1-\sin \left(S k_{a}(i) \times \frac{\pi}{2}\right)\right]
$$

where MT refers to the maximum threshold all agents can possess for a task. This creates a genetic stable point for agents, based on skill levels. Also they used the following formula for the amount of inhibition agent $a$ will give to another interacting agent:

$$
S k_{a}(i) \times I R \times M T
$$

assuming that inhibition was determined by agent's skill levels only. The influence rate $I R$ is a parameter that determines the strength of an agent's influence. This parameter can be universal or variable for each agent. It is also possible that influence rate can be different for each task. If $I R$ is taken to be dependent on the age of an agent, we can recreate the effect of polytheism. The authors [13] took $I R=0.5$ in formula (2) to study the effect of inhibition on their model. Though they improved the quality of work (QOW) but the levels of division of labor (DOL) was lowered.

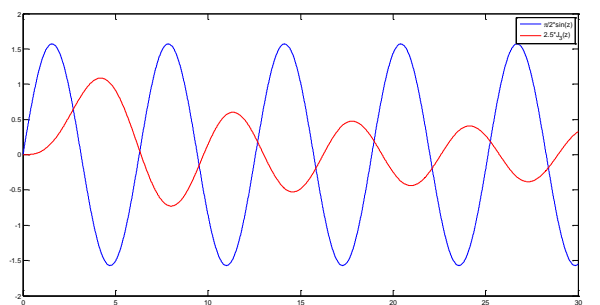

Figure 1a: Graph of Sin and Bessel function of 3rd kind

The reason for lower levels of division of labor in the model [13] is rather lower values of the genetic pull governed by equation (1). So, in our model, we have selected Bessel function because they behave like damped sin and cosine curves and stabilize over a longer period of time as evident from Figure 1a. This is because in the beginning of the simulation, agents have high potential to perform the task but as time passes energy levels will be lowered.

We constructed the following formula for genetic pull:

$$
M T \times\left[1-2.5 J_{3}\left(4.2 S k_{a}(i)\right)\right]
$$

The third order Bessel function $J_{3}$ was empirically selected instead of $J_{1}, J_{2}$ as it improved values of division of labor while maintaining an upper edge over the quality of work compared to model in [13]. We attribute this to the lower amplitude and flatter nature of the curve associated with $J_{3}$.

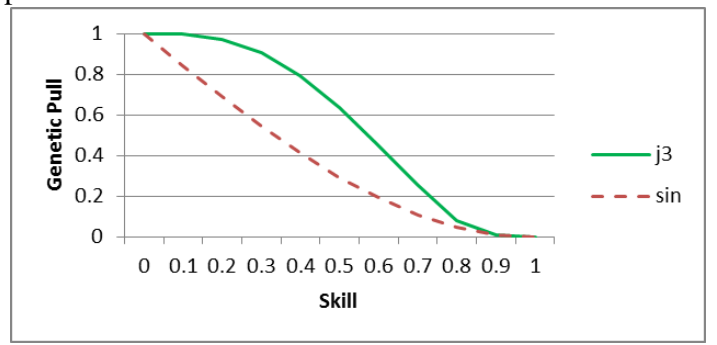

Figure 1b: Genetic pull vs. skill level

The Figure $1 \mathrm{~b}$ represents the graphs of the genetic pull governed by equations (1) (dashed line) and (3) (smooth line), taking the value of $M T=1$. From Figure $1 b$, it is seen that the genetic pull controlled by equation (3) has higher values than the one given by equation (1). As a consequence of this, we expect a better level of specialization which is indeed achieved.

\subsection{Stimulus Intensity}

Each time a task $i$ is performed by an individual, the stimulus intensity $S_{i}$, is decreased by an amount $\alpha=3$, (same as in [8]). For each time step, the level of the stimulus $S_{i}$, associated to task $j$ is increased by:

$$
\beta_{i}=\alpha \frac{N}{T} \delta
$$

where $N$ is the group size (number of individuals), $T$ the task number and $\delta$ the demand. Demand represents the total colony effort required to complete all tasks relative to the available total effort from workers. In [8], the authors fixed the demand for all tasks thus; the rate of stimulus regeneration is identical for all tasks and does not vary over time.

We believe this is not very realistic, so the rate of stimulus regeneration is allowed to vary over time. This is achieved by considering the demand $\delta$ varying continuously over a smooth curve as shown in Figure 2. The curve is generated by using the following formula:

$$
0.5+1.2 J_{3}\left(\frac{25 t}{1000}-2\right)
$$

where $t$ is the simulation time step. Each simulation lasted 1000 time steps. The change in demand, in general, is oscillatory in nature and stabilizes over a longer period of time. This motivated us to choose the formula (5) for varying the demand with time satisfying both the requirements.

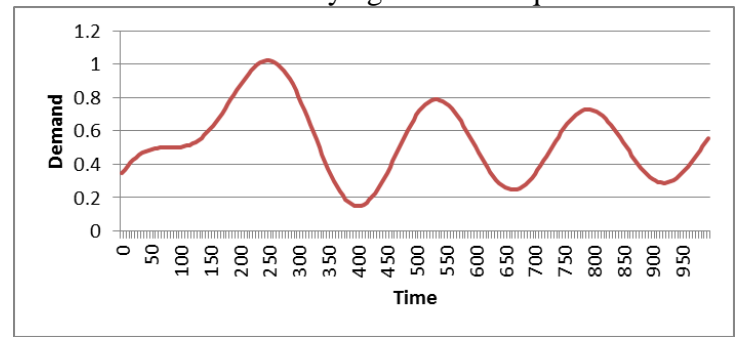

Figure 2: Demand vs. Time

This continuous choice of demand has the advantage that in each time step, the stimulus changes thus depicting the real world more accurately. Demand is chosen randomly in $(0.1$, 1.1), for each task. In this case each task has a different stimulus which was omitted for simplicity in [8].

Further to compare our results with that of [8], we choose the same discrete values of $\delta$ as in [8].

\section{EXPERIMENTS AND RESULTS}

\subsection{Design of experiments}

The main focus of this section is to design experiments to observe the influence of (i) demand $\delta=0.7,0.9,1.0,1.1$, (ii) demand chosen randomly in $(0.1,1.1)$ and (iii) demand varying continuously over the smooth oscillatory curve of figure (2); on DOL and QOW.

A metric to measure level of specialization within a population was developed in [14]. The same metric to measure DOL is used. The measure quantifies the degree to which agents in a population are specialized. Each agent records their task allocation amounts. These amounts are then stored in an $n \times m$ matrix, where $n$ is the number of agents and $m$ is the number of tasks. Then this matrix is normalized 
such that the sum of all cells is 1 . The mutual information and Shannon entropy index [15] are then calculated for the distribution of individuals across tasks. Finally, dividing the mutual information score by the Shannon entropy score will provide a value between 0 and 1 [12]. A score of 0 indicates a population with no specialization and a score of 1 indicates a fully specialized population [14].

The metric developed in [13] to measure quality of work (QOW) is used. It is a measure of the average amount of skill used in performing a task. The quality of work is a value between 0 and 1 . A higher value indicates that the task was performed by a more skilled agent. All the agents are assigned an average skill level of 0.5 .

Agents will perform one of tasks that cross its thresholds or be inactive if no such task exits. Each individual was given a uniformly random initial threshold value for each task between 0 and 3, which served as the maximum threshold. Each agent was also given a random skill level between 0 and 1 for each task.

Simulations were run for 100 times for each combination of the parameters. The models were compared across several combinations of tasks and agent counts. Similar to the original paper, the simulations with run with 2, 4, 10 and 20 tasks and 10, 50, 100, 500 and 1000 agents. For each combination, the resulting level of division of labor (DOL) and quality of work (QOW) were measured. The average values were then considered for a particular combination. The results are illustrated in the Figures 3-10. Each graph illustrates the values of DOL and QOW for the genetic pulls governed, respectively, by sin curve and by the proposed Bessel curve. The Y-axis of each graph presents the value between 0 and 1 . The $\mathrm{X}$ - axis represents each level of agent count that we used.

\subsection{Comparison with existing model}

In this section, the level of specialization between this model and the one proposed in [13] are compared. The effect of the new formula (3) for genetic pull is reflected in the Figures 36 , where $J_{3}$ (diamond) and $\sin$ (square) represent DOL from this model and from the one proposed in [13]. Better values of DOL are achieved as compared to [13]. There is a general increase in the level of specialization as the agent count increases and also as the number of tasks increase.

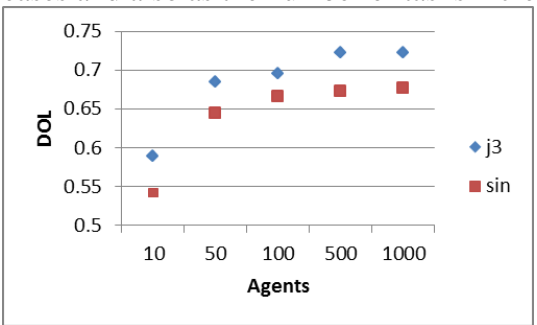

Figure 3: DOL with 2 tasks

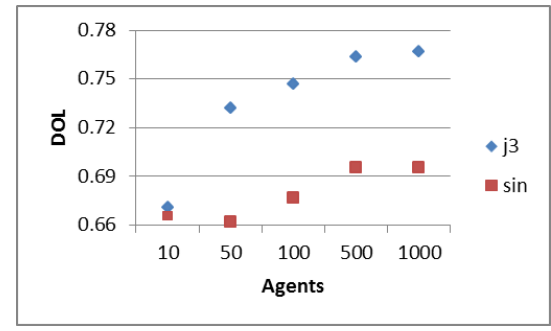

Figure 4: DOL with 4 tasks

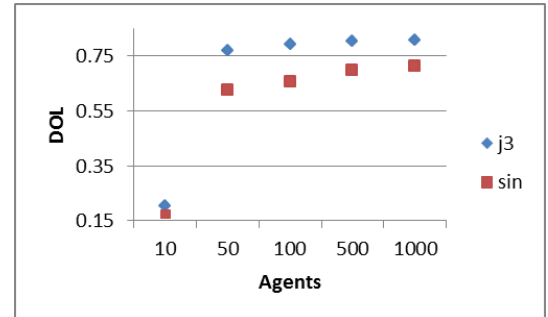

Figure 5: DOL with 10 tasks

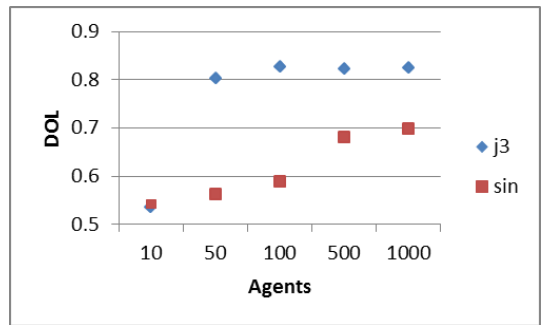

Figure 6: DOL with 20 tasks

In this model DOL always increases with increase in task count except when the number of tasks and agents were equal. The QOW is similar in the models proposed by us and in [13], and hence was omitted from the results.

\subsection{Discrete demand}

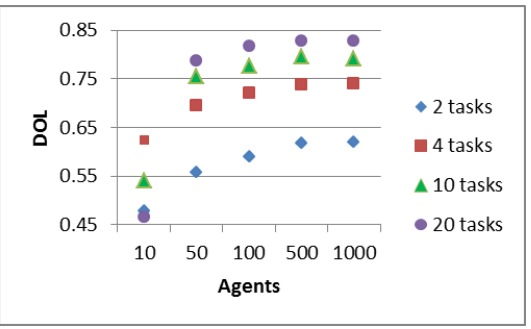

Figure 7: DOL with $\delta=0.7$

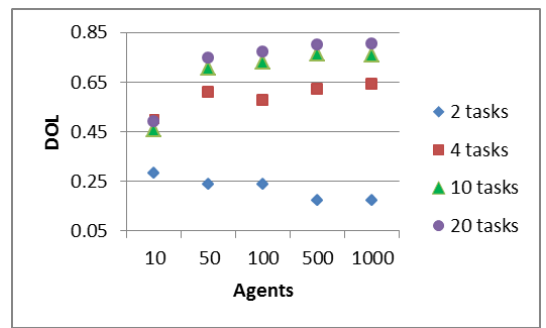

Figure 8: DOL with $\delta=0.9$

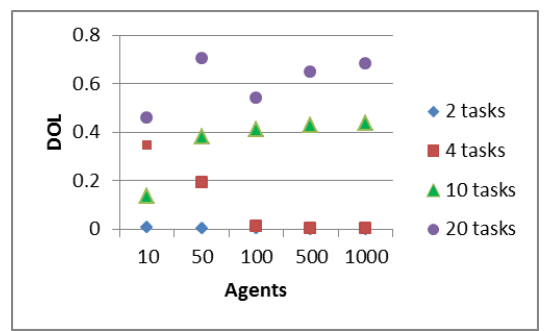

Figure 9: DOL with $\delta=1.0$ 


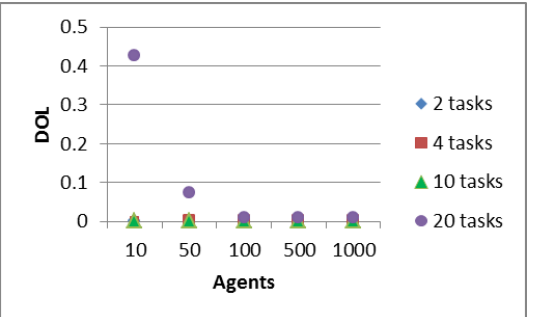

Figure 10: DOL with $\delta=1.1$

For $\delta=0.7$, DOL increases with group size for all tasks and for groups size 50 or more it increases with number of tasks. As demand increases to 1 , DOL decreases with group size for 2 and 4 tasks. For $\delta>1$, DOL drops as expected.

\subsection{Random demand}

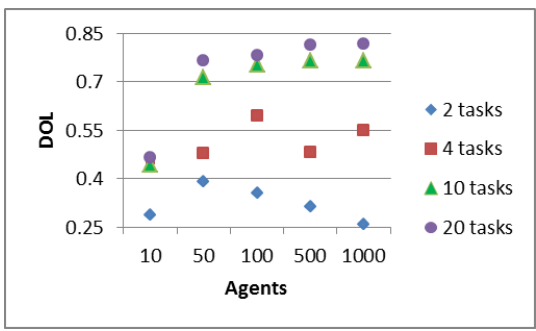

Figure 11: DOL with random demand

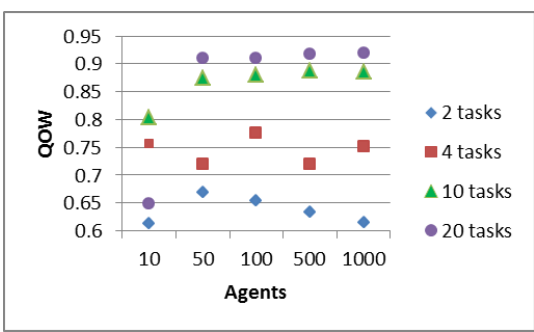

Figure 12: QOW with random demand

The level of specialization increases with tasks for random demand. For 10 and 20 tasks, the DOL increases with agent count. For 2 tasks, DOL increases with agent count till 50 agents and then starts decreasing with agent count. For 4 tasks, DOL oscillates between 0.45 and 0.6. The QOW follows similar pattern.

\subsection{Continuously varying demand}

The level of specialization increases monotonically with group size, except for 4 tasks, where there is a dip in specialization level for 10 agents. For all tasks the DOL stabilizes around 500 agents while QOW stabilizes around 50 agents. For population less than 50 agents, the QOW decreases with increase in task number.

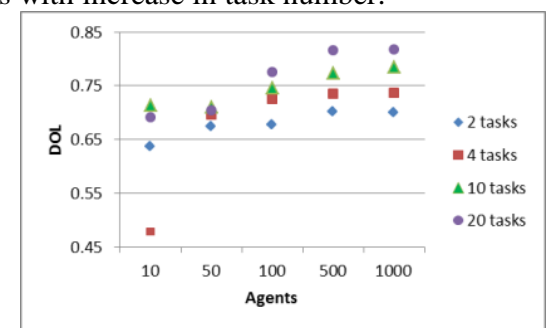

Figure 13: DOL with continuous demand

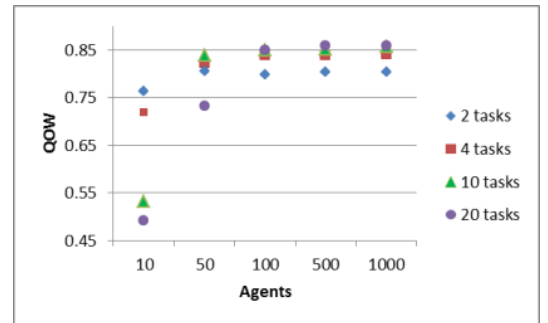

Figure 14: QOW with continuous demand

\section{DISCUSSION}

In the proposed model, specialization is influenced by number of agents, task number, and demand. There is interplay between these three parameters. The effect of task number and group size on DOL varies as demand moves above or below one. When demand is greater than one, from Eq.(4), it is seen that the stimulus intensity of each task rises quickly above the threshold of any agent so that all agents become equally likely to perform any task at each time step, regardless of thresholds. Hence, there is no proper division of labor (Figure 10). For demand equal to one, division of labor actually decreased with increasing group size for 2 and 4 tasks but it increased with group size for 10 and 20 tasks as illustrated by Figure 9 . When demand level is below 1, agents have fewer specializations that will have enough stimuli to surpass their thresholds. The results indicate that even when there is low demand, enough agents are still faced with multiple choices, resulting in a specialization from social influence. The role of a high task number for DOL is less if task number is 4 or more.

From the Figures 3 to 6 significant increases in division of labor in the new model compared to [13] is observed. The specialization increases monotonically with group size for 2 and 4 tasks whereas for 10 and 20 tasks, the DOL increases initially with group size and almost stabilizes for groups of size 100 or more.

The Figures 7 to 14, showing all the three cases of demand, imply that continuous demand is the better choice followed by random demand and then discrete demand at the bottom. QOW also follows the similar pattern in all the three cases.

\section{CONCLUSION AND FUTURE WORK}

We have proposed a new hybrid model by introducing a new formula for genetic pull. This helps to increase the DOL as compared with the model proposed in [13]. The QOW is either slightly better or at par with QOW achieved in [13]. The novelty of our approach is that we analyze the DOL and QOW by introducing the concept of continuous and random demand in our model. The demand changes depending on several factors like colony size, climatic changes across social systems as well as other biological systems. Assuming the food is the resource in an ant colony, its availability is higher during summer thus reducing the demand and consequently during winter demand is higher owing to scarcity of food. Hence demand is oscillatory in nature. Over a longer period, the colony also tries to preserve some food for leaner periods, hence the demand eventually stabilizes. Keeping these requirements in mind, we constructed formula (5) for the demand, which is both oscillatory and eventually stabilizes around a point.

The increase in the DOL with group size, as shown by Figures 3 to 6 , is in conformity with [6] and [7] who concluded that an increase in division of labor could parallel an increase in group size directly via the distribution of thresholds within groups and indirectly via by-products of increased group size (i.e. task number and demand). 
There is a marked difference in DOL and QOW for random demand compared to continuous demand. In the random case, both DOL and QOW are heavily task dependent. These are better for higher number of tasks as compared to fewer tasks. In the future work we will focus on a combination of our continuous and random approaches such that demand for each task varies continuously on a randomly chosen smooth curve.

\section{REFERENCES}

[1] Spencer, A. J., Couzin, I. D., and Franks, N. The dynamics of specialization and generalization within biological popula-tions, Journals of Complex Systems 1: 115-127 (1998)

[2] Maynard Smith, J., and Szathmary., E. The major Transitions in Evolution. Oxford University Press (1995)

[3] Oster, G. F., and Wilson, E. O. Caste and Ecology in the Social Insects. Princeton, NJ.: Princeton Univ. Press (1978)

[4] Bourke, A.F.G. Colony Size, Social complexity and reproductive conflicts in social insects. J. Evol. Biol 12: 245-257 (1999)

[5] Anderson C. and McShea, D.W. Individual versus social complexity, with particular references to ant colonies. Biological Reviews 76:211-237 (2001)

[6] Karsai, I., and Wenzel, J. W. Productivity, individuallevel and colony level flexibility, and organization of work as con-sequences of colony size. Proceeding of the National Academy of Sciences 95(15), 8665-8669 (1998)

[7] Thomas, M. L., and Elgar, M. A. Colony size affects division of labour in the ponerine ant Rhytidoponerametallica. Na-turwissenschaften 90: 88-92 (2003)
[8] Jeanson, R., Fewell, J. H., Gorelick, R., and Bertram, S.M. Emergence of increased division of labour as a function of group size. Behavioural Ecology and Sociobiology 62: 289-298 (2007)

[9] Beshers, S. N., and Fewell, J. H. Models of division of labour in social insects. Annual Review Entomology 46: 413-440 (2001)

[10] Traniello, J. F. A., and Rosengaus, R. B. Ecology, evolution and division of labour in social insects, Animal behaviour 53: 209-213 (1997)

[11] Cockburn, D., Kobti, Z. Wasps: A weight allocated social pressure systems for the emergence of agent specialization. Proceedings of the 20th European Conference on Artificial Life 161-167. MIT press (2011)

[12] G. Theraulaz, E. Bonabeau, and J.L. Deneubourg. Response threshold reinforcements and division of labor in insects so-cieties. In Proc. R.Soc.Lond.B.Biol.Sci., volume 265, pages 327-332, (1998).

[13] Cockburn, D., Kobti, Z. A Genetic and Social Computational Model for the Emergence of Skill Based Agent Specialization. Proceedings of the Twenty Fifth International Canadian Conference on AI 287-292 (2012)

[14] Gorelick, R., Bertram, S.M., and Killeen, P.R., Fewell, J.H. Normalized mutual entropy in biology: quantifying division of labor. American Naturalist 164: 678-682 (2004)

[15] C.E. Shannon. A mathematical theory of communication. Bell System Technical Journal, 23:379-423, 1948. 